\title{
4 GlueVaR and other new risk measures
}

This chapter is structured in two parts. Analytical closed-form expressions of GlueVaR risk measures for commonly used statistical distributions in the insurance context are derived. These closed-form expressions should enable practitioners to undertake an effortless transition from the use of VaR and TVaR to GlueVaR. Third order Cornish-Fisher approximations to GlueVaR risk measures for general skewed distribution functions are also introduced in this chapter. Finally, the relationship between GlueVaR, Tail Distortion risk measures and RVaR risk measures are shown.

\subsection{Analytical closed-form expressions of GlueVaR}

A useful consequence of (3.4) is that when analytical closed-form expressions of $\operatorname{VaR}_{\alpha}(X)$ and $\operatorname{TVaR}_{\alpha}(X)$ are known for a random variable $X$, the closed-form expression of GlueVaR ${ }_{\beta, \alpha}^{h_{1}, h_{2}}(X)$ can automatically be derived without further complications. Otherwise, using the definition of GlueVaR as a distortion risk measure, the Choquet Integral of $X$ with respect to the set function $\kappa_{\beta, \alpha}^{h_{1}, h_{2}} \circ P$ should be calculated.

\subsubsection{Illustration: GlueVaR expression for Student $t$ distribution}

Let $X$ be a random variable such that $\widetilde{X}=\frac{X-\mu}{\sigma}$ is distributed as a Student $t$ random variable with $v$ degrees of freedom (df). In such a case, $X$ has $\mu$ mean and a standard deviation equal to $\sqrt{\frac{v \cdot \sigma^{2}}{v-2}}$. Then

$$
\begin{gathered}
\operatorname{VaR}_{\alpha}(X)=\mu+\sigma \cdot t_{\alpha} \\
\operatorname{TVaR}_{\alpha}(X)=\mu+\sigma \cdot \frac{\tau\left(t_{\alpha}\right)}{1-\alpha} \cdot\left(\frac{v+t_{\alpha}^{2}}{v-1}\right),
\end{gathered}
$$


where $t_{\alpha}$ is the $\alpha$-quantile of a Student $t$ distribution with $v \mathrm{df}$ and $\tau$ is its density function.

Using (3.4) the GlueVaR of random variable $X$ is

$$
\begin{aligned}
\operatorname{GlueVaR}_{\beta, \alpha}^{h_{1}, h_{2}}(X) & =\omega_{1} \cdot\left[\mu+\sigma \cdot \frac{\tau\left(t_{\beta}\right)}{1-\beta} \cdot\left(\frac{v+t_{\beta}^{2}}{v-1}\right)\right] \\
+\omega_{2} \cdot\left[\mu+\sigma \cdot \frac{\tau\left(t_{\alpha}\right)}{1-\alpha} \cdot\left(\frac{v+t_{\alpha}^{2}}{v-1}\right)\right]+\left(1-\omega_{1}-\omega_{2}\right) \cdot\left(\mu+\sigma \cdot t_{\alpha}\right) & \\
= & \mu+\sigma \cdot\left[\left(\frac{h_{1}}{1-\beta}-\frac{h_{2}-h_{1}}{\beta-\alpha}\right) \cdot \tau\left(t_{\beta}\right) \cdot\left(\frac{v+t_{\beta}^{2}}{v-1}\right)\right. \\
& \left.+\frac{h_{2}-h_{1}}{\beta-\alpha} \cdot \tau\left(t_{\alpha}\right) \cdot\left(\frac{v+t_{\alpha}^{2}}{v-1}\right)+\left(1-h_{2}\right) \cdot t_{\alpha}\right]
\end{aligned}
$$

\subsubsection{Analytical expressions for other frequently used distributions}

Normal $(\mathscr{N})$, Lognormal $(\mathscr{L} \mathscr{N})$ and Generalized Pareto $(\mathscr{G} \mathscr{P})$ distributions have simple closed-form expressions of GlueVaR. The same notation conventions that were introduced in Chapter 1 are used. Namely, $\phi$ and $\Phi$ stand for the standard Normal pdf and cdf, respectively. The standard Normal distribution $\alpha$ and $\beta$ quantiles are denoted as $q_{\alpha}=\Phi^{-1}(\alpha)$ and $q_{\beta}=\Phi^{-1}(\beta)$. For the $\mathscr{G} \mathscr{P}$ distribution, the definition provided in Hosking and Wallis [1987] is considered, where the scale parameter is denoted by $\sigma$ and $k$ is the shape parameter. The $\mathscr{G} \mathscr{P}$ distribution contains the Uniform $(k=1)$, the Exponential $(k=0)$, the Pareto $(k<0)$ and the type II Pareto $(k>0)$ distributions as special cases. Closed-form expressions of GlueVaR for several distributions are presented in Table 4.1. Note that there are some exceptions to the general rule to deduce these closed-form expressions to be considered. When $X$ follows a Pareto distribution with $k \leqslant 1$ and for any confidence level $\alpha$, $\operatorname{TVaR}_{\alpha}(X)=+\infty$ as we have shown in Table 1.3 of Chapter 1. But when $h_{1}=0 \mathrm{GlueVaR}_{\beta, \alpha}^{h_{1}, h_{2}}(X)$ is finite. There is a compensation effect between $\operatorname{TVaR}_{\alpha}(X)$ and $\operatorname{TVaR}_{\beta}(X)$. This is taken into account in Table 4.1. 
Table 4.1 Closed-form expressions of GlueVaR for some selected distributions

\begin{tabular}{|c|c|}
\hline $\begin{array}{l}\text { Distribu- } \\
\text { tion }\end{array}$ & GlueVaR $_{\beta, \alpha^{h_{1}, h_{2}}}$ expression \\
\hline $\begin{array}{l}\text { Normal: } \\
\qquad \mathscr{N}\left(\mu, \sigma^{2}\right)\end{array}$ & $\begin{array}{l}\mu+\sigma \cdot q_{\alpha} \cdot\left(1-h_{2}\right)+\sigma \cdot \frac{h_{2}-h_{1}}{\beta-\alpha} \cdot\left[\phi\left(q_{\alpha}\right)-\phi\left(q_{\beta}\right)\right]+\sigma \cdot \frac{h_{1}}{1-\beta} \\
\phi\left(q_{\beta}\right)\end{array}$ \\
\hline $\begin{array}{l}\text { Lognormal: } \\
\qquad \mathscr{L} \mathscr{N}\left(\mu, \sigma^{2}\right)\end{array}$ & $\begin{array}{l}\exp \left(\mu+\sigma \cdot q_{\alpha}\right) \cdot\left(1-h_{2}\right) \\
+\exp \left(\mu+\frac{\sigma^{2}}{2}\right) \cdot \frac{h_{2}-h_{1}}{\beta-\alpha} \cdot\left[\Phi\left(\sigma-q_{\alpha}\right)-\Phi\left(\sigma-q_{\beta}\right)\right] \\
+\exp \left(\mu+\frac{\sigma^{2}}{2}\right) \cdot \frac{h_{1}}{1-\beta} \cdot \Phi\left(\sigma-q_{\beta}\right)\end{array}$ \\
\hline $\begin{array}{l}\text { Exponen- } \\
\text { tial: } \\
\qquad \mathscr{G} \mathscr{P}(k, \sigma) \\
\text { with } k=0\end{array}$ & $\sigma \cdot\left[h_{2}-\ln (1-\alpha)\right]+\sigma \cdot(1-\beta) \cdot \ln \left(\frac{1-\beta}{1-\alpha}\right) \cdot\left[\frac{h_{2}-h_{1}}{\beta-\alpha}-\frac{h_{1}}{1-\beta}\right.$ \\
\hline
\end{tabular}


Table 4.1: continued from previous page

\begin{tabular}{|c|c|}
\hline $\begin{array}{l}\text { Distribu- } \\
\text { tion }\end{array}$ & GlueVaR $_{\beta, \alpha^{h_{1}, h_{2}}}$ expression \\
\hline $\begin{array}{l}\text { Pareto: } \\
\qquad \mathscr{G} \mathscr{P}(k, \sigma) \\
\text { with } k<0\end{array}$ & 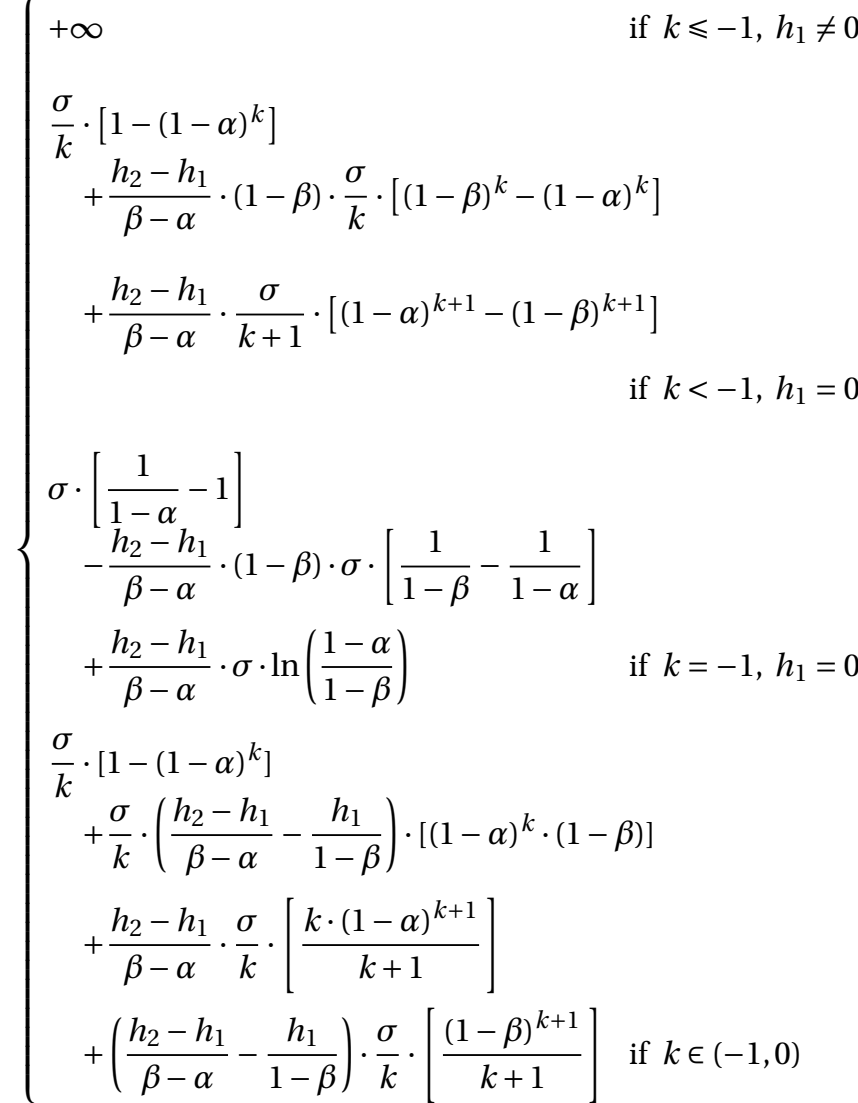 \\
\hline $\begin{array}{l}\text { Type II } \\
\text { Pareto: } \\
\mathscr{G} \mathscr{P}(k, \sigma) \text {, } \\
\text { with } k>0\end{array}$ & $\begin{array}{l}\frac{\sigma}{k} \cdot\left[1-(1-\alpha)^{k}\right]+\frac{\sigma}{k} \cdot\left(\frac{h_{2}-h_{1}}{\beta-\alpha}-\frac{h_{1}}{1-\beta}\right) \cdot\left[(1-\alpha)^{k} \cdot(1-\beta)\right] \\
+\frac{h_{2}-h_{1}}{\beta-\alpha} \cdot \frac{\sigma}{k} \cdot\left[\frac{k \cdot(1-\alpha)^{k+1}}{k+1}\right] \\
+\left(\frac{h_{2}-h_{1}}{\beta-\alpha}-\frac{h_{1}}{1-\beta}\right) \cdot \frac{\sigma}{k} \cdot\left[\frac{(1-\beta)^{k+1}}{k+1}\right]\end{array}$ \\
\hline
\end{tabular}

\subsubsection{The Cornish-Fisher approximation of GlueVaR}

General considerations about Cornish-Fisher VaR methodologies have been pointed out in Section 1.2.2 of Chapter 1. Approximations to GlueVaR risk 
measures for general skewed distribution functions using a Cornish-Fisher expansion of their quantiles are provided in this section. In insurance applications managers often have to face to highly skewed random variables with right fat tails. In many of these situations, however, they do not know whether the underlying random variable of interest is distributed according to a known parametric distribution function. In those situations that the distribution is unknown, the value of the common quantile-based risk measures is routinely approximated by practitioners. It is shown that approximations of GlueVaR risk measures for general unknown skewed distribution functions can be directly obtained by means of the relationship of GlueVaR risk measures and the standard quantile-based risk measures.

The Cornish-Fisher expansion is widely used by practitioners to approximate the $\operatorname{VaR}_{\alpha}(X)$ and $\operatorname{TVaR}_{\alpha}(X)$ values when the random variable follows a skewed unknown distribution [see Cornish and Fisher, 1937; Fisher and Cornish, 1960; Johnson and Kotz, 1970; McCune and Gray, 1982]. The VaR and TVaR measure values can be approximated as $\operatorname{VaR}_{\alpha}(X) \simeq \mu+q_{v, \alpha} \sigma$ and $\operatorname{TVaR}_{\alpha}(X) \simeq \mu+q_{t v, \alpha} \sigma$, where $\mu=\mathbb{E}[X], \sigma^{2}=\mathbb{V}[X]$ and both $q_{v, \alpha}$ and $q_{t v, \alpha}$ are modified quantiles of the standard Normal distribution that take into account the skewness of the distribution function of $X$.

Following Sandström [2007], the modified quantiles $q_{v, \alpha}$ and $q_{t v, \alpha}$ are computed as follows. Let us consider $\gamma=\mathbb{E}\left[(X-\mu)^{3}\right] / \sigma^{3}$ as a measure of the skewness of the random variable $X$. If $q_{\alpha}=\Phi^{-1}(\alpha)$ and $\phi$ are the $\alpha$-quantile and the density function of the standard Normal distribution, respectively, then $q_{v, \alpha}$ and $q_{t v, \alpha}$ can be written as,

$$
\begin{aligned}
q_{v, \alpha} & =\Phi^{-1}(\alpha)+\frac{\gamma}{6}\left[\left(\Phi^{-1}(\alpha)\right)^{2}-1\right]=q_{\alpha}+\frac{\gamma}{6}\left[q_{\alpha}^{2}-1\right] \\
q_{t v, \alpha} & =\frac{\phi\left(\Phi^{-1}(\alpha)\right)}{1-\alpha}\left[1+\frac{\gamma}{6}\left(\Phi^{-1}(\alpha)\right)^{3}\right]=\frac{\phi\left(q_{\alpha}\right)}{1-\alpha}\left[1+\frac{\gamma}{6} q_{\alpha}^{3}\right] .
\end{aligned}
$$

Extensions of the Cornish-Fisher expansion that consider moments of higher order than $\gamma$ have been provided in the literature [see, for instance, Giamouridis, 2006]. More details can be found in Appendix B of Sandström [2011].

Given the interpretation of a GlueVaR risk measure as a linear combination of risk measures which was shown in (3.4), the approximation for the GlueVaR of the random variable $X$ following the Cornish-Fisher expansion can 
be obtained as

$$
\begin{aligned}
& \operatorname{GlueVaR}_{\beta, \alpha}^{h_{1}, h_{2}}(X) \simeq \mu+\sigma\left[\left(\frac{h_{1}}{1-\beta}-\frac{h_{2}-h_{1}}{\beta-\alpha}\right) \phi\left(q_{\beta}\right)\left(1+\frac{\gamma}{6} q_{\beta}^{3}\right)\right. \\
& \left.+\left(\frac{h_{2}-h_{1}}{\beta-\alpha}\right) \phi\left(q_{\alpha}\right)\left(1+\frac{\gamma}{6} q_{\alpha}^{3}\right)+\left(1-h_{2}\right)\left(\frac{\gamma}{6}\left(q_{\alpha}^{2}-1\right)+q_{\alpha}\right)\right] .
\end{aligned}
$$

The error of the approximation is upper bounded by the maximum error incurred when approximating $\operatorname{VaR}_{\alpha}(X)$, $\operatorname{TVaR}_{\alpha}(X)$ and $\operatorname{TVaR}_{\beta}(X)$ using the equivalent Cornish-Fisher expansion for skewed distributions. This result is straightforward. It follows from the linear relationship shown in expression (3.4) and taking into account that weights $\omega_{1}, \omega_{2}$ and $\omega_{3}$ are lower or equal than one, satisfying that $\omega_{1}+\omega_{2}+\omega_{3}=1$.

\subsection{On the relationship between GlueVaR and Tail Distortion risk measures}

As it has been aforementioned, different works that pay attention to risk measures based on distortion functions or based on several generalizations of quantiles have appeared in recent years. See, for instance, Zhu and Li [2012]; Bellini and Gianin [2012]; Bellini et al. [2014]; Dhaene et al. [2012a] and Goovaerts et al. [2012].

This section is devoted to reveal the connections between GlueVaR risk measures and Tail Distortion risk measures. To the best of our knowledge, Tail Distortion risk measures were introduced in Zhu and Li [2012]. Here the notation used for these family of risk measures is adapted from that in Lv et al. [2013]. Consider a distortion function $g$, this is a non-decreasing and injective function $g$ from $[0,1]$ to $[0,1]$ such that $g(0)=0$ and $g(1)=1$, and a confidence level $\alpha \in(0,1)$. The Tail Distortion Risk Measure $\mathrm{T}_{g, \alpha}$ associated to $g$ and $\alpha$ is defined as the distortion risk measure with distortion function $g_{\alpha}$, where

$$
g_{\alpha}(u)=g\left(\frac{u}{1-\alpha}\right) \cdot \mathbb{1}[0 \leqslant u<1-\alpha]+\mathbb{1}[1-\alpha \leqslant u \leqslant 1] .
$$

Note that $\mathbb{1}[0 \leqslant u<1-\alpha]$ is a function that takes value 1 in the interval $[0 \leqslant u<1-\alpha]$ and 0 elsewhere. In other words, if $X$ is a random variable representing a loss in a probability space $(\Omega, \mathscr{A}, P)$ and its survival function is $S_{X}(x)=P(X>x)$, then

$$
\mathrm{T}_{g, \alpha}(X)=\int_{-\infty}^{0}\left[g_{\alpha}\left(S_{X}(x)\right)-1\right] d x+\int_{0}^{+\infty} g_{\alpha}\left(S_{X}(x)\right) d x .
$$


Note that $g_{\alpha}$ is continuous in $1-\alpha$ or, alternatively, $g_{\alpha}(1-\alpha)=1$.

Proposition 4.1. Consider a GlueVaR $\omega_{\beta, \alpha}^{\omega_{1}, \omega_{2}}$ risk measure with parameters $\alpha$, $\beta, \omega_{1}$ and $\omega_{2}$. This GlueVaR is equivalent to a Tail Distortion riskmeasure $\mathrm{T}_{g, \alpha}$ if, and only if, $\omega_{2}=1-\omega_{1}$ and

$$
\begin{aligned}
g(t)=\left(\frac{\omega_{1} \cdot(1-\alpha)}{1-\beta}+1-\omega_{1}\right) \cdot t \cdot \mathbb{1}\left[0 \leqslant t<(1-\alpha)^{-1} \cdot(1-\beta)\right] \\
+\left(\omega_{1}+\left(1-\omega_{1}\right) \cdot t\right) \cdot \mathbb{1}\left[(1-\alpha)^{-1} \cdot(1-\beta) \leqslant t \leqslant 1\right] .
\end{aligned}
$$

The proof is provided in Section A.3 of the Appendix.

Note that only GlueVaR risk measures with $\omega_{3}=0$ can be represented as Tail Distortion risk measures, because $\omega_{1}+\omega_{2}+\omega_{3}=1$ must hold as part of the definition of a GlueVaR $\omega_{\beta, \alpha}^{\omega_{1}, \omega_{2}}$ risk measure. In other words, one can only represent as Tail Distortion risk measures those GlueVaR that do not weight the part corresponding to the $\operatorname{VaR}_{\alpha}$.

The origin of GlueVaR risk measures can be found in Belles-Sampera [2011]. As a curiosity, the definition of a parametric family of risk measures named PUp-TVaR can also be found therein, which are exactly the Tail Distortion risk measures linked to Proportional Hazards Distortion functions $g(u)=$ $u^{\frac{1}{a}}, a \geqslant 1$ from the perspective of Zhu and $\mathrm{Li}$ [2012].

\subsection{On the relationship between GlueVaR and RVaR risk measures}

To the best of our knowledge RVaR risk measures were introduced in Cont et al. [2010]. This section is dedicated to show a close relationship between GlueVaR risk measures and the RVaR family. We have recently discovered this connection. It is highly probable that some synergies between the research associated to both families are going to arise. For instance, existing results related to capital allocation principles using RVaR [see, for instance, Embrechts et al., 2016] and the ones obtained with GlueVaR (which we are going to present in Chapter 7) may be interconnected. Another example of these synergies may be found in the analysis of risk attitudes that we present in Chapter 5 because, as we will show, the application of our assessment tools are straightforward in RVaR risk measures.

Let $X$ be an absolutely continuous random variable, which positive values represent losses. Let $\alpha, \beta \in[0,1]$. The value of the risk measure $\operatorname{RVaR}_{\alpha, \beta}$ 
applied to $X$ is, by definition, the following:

$$
\operatorname{RVaR}_{\alpha, \beta}(X)= \begin{cases}\frac{1}{\beta} \int_{\alpha}^{\alpha+\beta} \operatorname{VaR}_{\gamma}(X) d \gamma & \text { if } \beta>0 \\ \operatorname{VaR}_{\alpha}(X) & \text { if } \beta=0\end{cases}
$$

The notation used for VaR is not the one used when defining GlueVaR risk measures, because $\operatorname{VaR}_{\alpha}(X)$ in (4.3) refers to the $100(1-\alpha) \%$ quantile while in GlueVaR definition the notation $\operatorname{VaR}_{\alpha}(X)$ is used to representing the $100 \alpha \%$ quantile. In order to find relationships between RVaR and GlueVaR risk measures it is convenient to rewrite expression (4.3) as

$$
\operatorname{RVaR}_{\alpha, \beta}(X)= \begin{cases}\frac{1}{\beta} \int_{\alpha}^{\alpha+\beta} \operatorname{VaR}_{1-\gamma}(X) d \gamma & \text { if } \beta>0 \\ \operatorname{VaR}_{1-\alpha}(X) & \text { if } \beta=0\end{cases}
$$

The notation used in definition of GlueVaR risk measures for VaR is incorporated in (4.4).

Note that

$$
\begin{aligned}
\frac{1}{\beta} \int_{\alpha}^{\alpha+\beta} \operatorname{VaR}_{1-\gamma}(X) d \gamma & =\frac{1}{\beta} \int_{1-(\alpha+\beta)}^{1-\alpha} \operatorname{VaR}_{\lambda}(X) d \lambda \\
& =\frac{1}{\beta}\left[\int_{1-(\alpha+\beta)}^{1} \operatorname{VaR}_{\lambda}(X) d \lambda-\int_{1-\alpha}^{1} \operatorname{VaR}_{\lambda}(X) d \lambda\right] \\
\text { notation used in def. of } & =\frac{1}{\beta}\left[(\alpha+\beta) \operatorname{TVaR}_{1-(\alpha+\beta)}(X)-\alpha \operatorname{TVaR}_{1-\alpha}(X)\right] \\
\text { GlueVaR } & =\frac{\alpha+\beta}{\beta} \operatorname{TVaR}_{1-(\alpha+\beta)}(X)-\frac{\alpha}{\beta} \operatorname{TVaR}_{1-\alpha}(X)
\end{aligned}
$$

Let us introduce some additional notation: $a=1-\alpha$ and $b=1-\beta$. So it can be deduced that $1-(\alpha+\beta)=a+b-1$ and that $\alpha+\beta=2-(a+b)$. Therefore, last expression in (4.5) may be rewritten as

$$
\frac{2-(a+b)}{1-b} \operatorname{TVaR}_{a+b-1}(X)-\frac{1-a}{1-b} \operatorname{TVaR}_{a}(X)
$$

Note now that $a+b-1 \leqslant a$ because $a, b \in[0,1]$, and this implies that $\operatorname{TVaR}_{a}(X) \geqslant \operatorname{TVaR}_{a+b-1}(X)$. Additionally, if

$$
\omega_{1}=-\frac{1-a}{1-b} \text { and } \omega_{2}=\frac{2-(a+b)}{1-b}
$$

then $\omega_{1}+\omega_{2}=\frac{1}{1-b}[a-1+2-a-b]=\frac{1-b}{1-b}=1$. 
Recall that a usual way to write the value of a GlueVaR risk measure applied to $X$ is as follows:

$$
\begin{aligned}
\operatorname{GlueVaR}_{\widehat{\beta}, \widehat{\alpha}}^{\widehat{h_{1}}, \widehat{h_{2}}}(X)=\widehat{\omega_{1}} \operatorname{TVaR}_{\widehat{\beta}}(X) & +\widehat{\omega_{2}} \operatorname{TVaR}_{\widehat{\alpha}}(X) \\
& +\left(1-\widehat{\omega_{1}}-\widehat{\omega_{2}}\right) \operatorname{VaR}_{\widehat{\alpha}}(X)
\end{aligned}
$$

where $\widehat{\omega_{1}}$ and $\widehat{\omega_{2}}$ depend on parameters $\widehat{\alpha}, \widehat{\beta}, \widehat{h_{1}}$ and $\widehat{h_{2}}$.

Taking into account the previous expressions and notations, consider

$$
\begin{aligned}
\widehat{\alpha} & =a+b-1 \\
\widehat{\beta} & =a \\
\widehat{\omega_{1}} & =\omega_{1}=-\frac{1-a}{1-b} \\
\widehat{\omega_{2}} & =\omega_{2}=\frac{2-(a+b)}{1-b} .
\end{aligned}
$$

As long as $\widehat{\omega_{1}}$ and $\widehat{\omega_{2}}$ are related to $\widehat{h_{1}}$ and $\widehat{h_{2}}$ by the next expression (as it is shown in Section A.2 of the Appendix)

$$
\left(\begin{array}{l}
\widehat{h_{1}} \\
\widehat{h_{2}}
\end{array}\right)=\left(\begin{array}{cc}
1 & \frac{1-\widehat{\beta}}{1-\widehat{\alpha}} \\
1 & 1
\end{array}\right)\left(\begin{array}{l}
\widehat{\omega_{1}} \\
\widehat{\omega_{2}}
\end{array}\right)
$$

it is deduced from (4.9) and (4.10) that

$$
\begin{aligned}
& \widehat{h_{1}}=-\frac{1-a}{1-b}+\left[\frac{2-(a+b)}{1-b}\right] \frac{1-a}{2-(a+b)}=\frac{-(1-a)+(1-a)}{1-b}=0 \\
& \widehat{h_{2}}=-\frac{1-a}{1-b}+\left[\frac{2-(a+b)}{1-b}\right]=\frac{1-b}{1-b}=1
\end{aligned}
$$

So, putting (4.8), (4.9) and (4.11) altogether the following expression holds:

$$
\begin{aligned}
\operatorname{GlueVaR}_{a, a+b-1}^{0,1}(X)=- & \frac{1-a}{1-b} \operatorname{TVaR}_{a}(X) \\
& +\frac{2-(a+b)}{1-b} \operatorname{TVaR}_{a+b-1}(X) .
\end{aligned}
$$

The right-hand side of expression (4.12) is exactly expression (4.6). This means that, for $\beta>0$ (or, equivalently, for $b<1$ ),

$$
\mathrm{RVaR}_{\alpha, \beta}=\mathrm{GlueVaR}_{a, a+b-1}^{0,1} .
$$

For $\beta=0$ (or $b=1)$, expression (4.12) is not well defined, but $a+b-1=a$ in such a case and it can be checked that GlueVaR ${ }_{a, a}^{0,1}=\operatorname{VaR}_{a}$. To get the feeling that this is correct, let us plot the distortion function of GlueVaR ${ }_{a, a+b-1}^{0,1}$ in Figure 4.1. 
Figure 4.1 Distortion function of GlueVaR ${ }_{a, a+b-1}^{0,1}$ distortion risk measure.

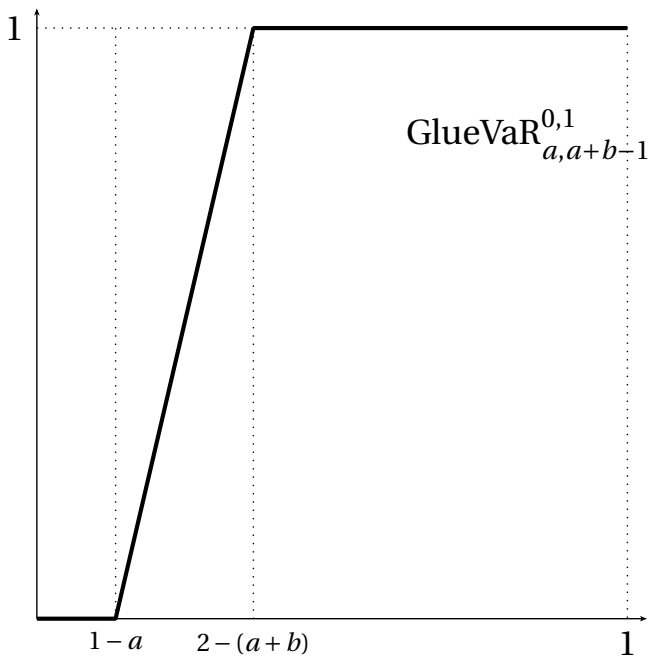

Looking at the plot of the distortion function in Figure 4.1, if $b=1$ then $2-(a+b)=1-a$ and then the distortion function of $\operatorname{VaR}_{a}$ would appear. In addition, if $a=1$ (which means that $\alpha=0$ ) then the distortion function of $\mathrm{TVaR}_{b}$ is obtained. As it is one of the purposes of $\mathrm{RVaR}_{\alpha, \beta}$ risk measures, it is possible to continuously moving from $\mathrm{VaR}_{1-\alpha}$ to $\mathrm{TVaR}_{1-\beta}$.

Taking all the previous comments into account, the following equivalence holds:

$$
\operatorname{RVaR}_{\alpha, \beta}=\mathrm{GlueVaR}_{a, a+b-1}^{0,1}
$$

\subsection{Example}

The example of risk quantification shown in Chapter 3 is followed to estimate GlueVaR risk measures considering alternative distributions. Outcomes are shown in Table 4.2. The table is divided into four blocks, each block representing the corresponding risk figures for the cost of claims for property damage $\left(X_{1}\right)$, the cost of claims of bodily injuries $\left(X_{2}\right)$, the cost of claims of medical expenses $\left(X_{3}\right)$ and the aggregate cost of claims $\left(X_{1}+\right.$ $X_{2}+X_{3}$ ). Risk measure values using the empirical distribution (first row) are compared with outcomes when Normal, Lognormal, Student $t$ with 4 $\mathrm{df}$ and Generalized Pareto distributions are fitted to data. In the last two rows of each block results are shown when risk measure values are approx- 
imated by a Cornish-Fisher expansion. The sample mean $(\widehat{\mu}=\bar{z})$, the sample deviation $\left(\widehat{\sigma}^{2}=\sum_{i}\left(Z_{i}-\bar{z}\right)^{2} /(n-1)\right)$ and the sample skewness (calculated as $\left.\widehat{\gamma}=\widehat{\sigma}^{-3}\left(\sum_{i}\left(Z_{i}-\bar{z}\right)^{3} / n\right)\right)$ are considered as estimators of $\mu, \sigma$ and $\gamma$ when $Z$ is one of the four random variables $X_{1}, X_{2}, X_{3}, X_{1}+X_{2}+X_{3}$. Sample statistics were computed using observations that fall below the $99.5 \%$ quantile in order to exclude the effect of extreme losses on estimates (first Cornish-Fisher approximation). That means, a subsample of the first 348 increasingly ordered elements of the random variable were used to estimate parameters. Therefore, the two highest values were considered as extreme losses and were not included. Outcome values of risk measures were compared with the risk measure approximations when all the observations are included on sample estimates (second Cornish-Fisher approximation). All the calculations were made in $\mathrm{R}$ and MS Excel.

Table 4.2 Examples of risk measurement of costs of insurance claims using quantile-based risk measures

\begin{tabular}{|c|c|c|c|c|c|c|}
\hline \multirow[b]{2}{*}{ Model } & \multirow[b]{2}{*}{ VaR $_{95 \%}$} & \multirow[b]{2}{*}{ TVaR $_{95 \%}$} & \multirow[b]{2}{*}{ TVaR $_{99.5 \%}$} & \multicolumn{3}{|c|}{ GlueVaR $_{\mathbf{9 9 . 5 \% , 9 5 \%}}^{h_{1}, h_{2}}$} \\
\hline & & & & $\left(\frac{11}{30}, \frac{2}{3}\right)$ & $(\mathbf{0}, \mathbf{1})$ & $\left(\frac{1}{20}, \frac{1}{8}\right)$ \\
\hline \multicolumn{7}{|l|}{$X_{1}$} \\
\hline Empirical & 2.5 & 12.5 & 40.8 & 18.6 & 9.4 & 4.9 \\
\hline Normal & 6.9 & 8.5 & 11.6 & 9.0 & 8.1 & 7.2 \\
\hline Lognormal & 2.7 & 8.7 & 32.7 & 14.5 & 5.4 & 4.4 \\
\hline Student $t$ (4 d.f.) & 8.8 & 12.8 & 24.6 & 15.4 & 11.5 & 9.8 \\
\hline Pareto & 2.5 & 5.5 & 18.1 & 8.7 & 4.1 & 3.4 \\
\hline Cornish-Fisher $^{(1 a)}$ & 8.5 & 27.8 & 128.4 & 54.9 & 16.6 & 15.1 \\
\hline Cornish-Fisher $^{(1 b)}$ & 16.3 & 59.1 & 284.0 & 119.8 & 34.1 & 31.0 \\
\hline \multicolumn{7}{|l|}{$X_{2}$} \\
\hline Empirical & 0.6 & 8.0 & 42.1 & 16.9 & 4.2 & 2.9 \\
\hline Normal & 6.0 & 7.4 & 10.2 & 7.9 & 7.1 & 6.3 \\
\hline Lognormal & 1.6 & 5.8 & 25.7 & 11.0 & 3.6 & 3.0 \\
\hline Student $t$ ( 4 d.f.) & 7.6 & 11.2 & 21.7 & 13.5 & 10.1 & 8.5 \\
\hline Pareto & 1.6 & 3.5 & 11.9 & 5.7 & 2.6 & 2.2 \\
\hline Cornish-Fisher $^{(2 a)}$ & 2.2 & 2.7 & 3.7 & 2.9 & 2.6 & 2.3 \\
\hline Cornish-Fisher ${ }^{(2 b)}$ & 6.1 & 7.5 & 10.3 & 8.0 & 7.2 & 6.4 \\
\hline \multicolumn{7}{|l|}{$X_{3}$} \\
\hline Empirical & 1.1 & 1.3 & 1.8 & 1.4 & 1.2 & 1.1 \\
\hline
\end{tabular}


Table 4.2: continued from previous page

\begin{tabular}{|c|c|c|c|c|c|c|}
\hline \multirow[b]{2}{*}{ Model } & \multirow[b]{2}{*}{$\operatorname{VaR}_{95} \%$} & \multirow[b]{2}{*}{ TVaR95\% } & \multirow[b]{2}{*}{ TVaR $_{99.5 \%}$} & \multicolumn{3}{|c|}{ GlueVaR $_{\mathbf{9 9 . 5 \% , 9 5 \%}}^{h_{1}, h_{2}}$} \\
\hline & & & & $\left(\frac{11}{30}, \frac{2}{3}\right)$ & $(\mathbf{0}, \mathbf{1})$ & $\left(\frac{1}{20}, \frac{1}{8}\right)$ \\
\hline Normal & 0.9 & 1.0 & 1.3 & 1.1 & 1.0 & 0.9 \\
\hline Lognormal & 0.8 & 1.4 & 3.2 & 1.8 & 1.2 & 1.0 \\
\hline Student $t$ ( 4 d.f.) & 1.0 & 1.4 & 2.5 & 1.7 & 1.3 & 1.1 \\
\hline Pareto & 0.9 & 1.4 & 3.0 & 1.8 & 1.2 & 1.0 \\
\hline Cornish-Fisher $^{(3 a)}$ & 1.0 & 2.1 & 7.6 & 3.6 & 1.5 & 1.4 \\
\hline Cornish-Fisher ${ }^{(3 b)}$ & 1.1 & 2.0 & 6.1 & 3.1 & 1.5 & 1.4 \\
\hline \multicolumn{7}{|l|}{$X_{1}+X_{2}+X_{3}$} \\
\hline Empirical & 5.9 & 19.7 & 81.1 & 35.6 & 12.9 & 10.2 \\
\hline Normal & 12.6 & 15.4 & 21.1 & 16.4 & 14.8 & 13.2 \\
\hline Lognormal & 5.5 & 15.8 & 60.7 & 27.3 & 10.8 & 8.6 \\
\hline Student $t$ (4 d.f.) & 16.0 & 23.2 & 44.4 & 27.8 & 20.8 & 17.7 \\
\hline Pareto & 5.0 & 11.0 & 36.0 & 17.3 & 8.1 & 6.7 \\
\hline Cornish-Fisher $^{(4 a)}$ & 11.0 & 34.3 & 155.5 & 66.9 & 20.8 & 19.0 \\
\hline Cornish-Fisher $^{(4 b)}$ & 34.6 & 134.0 & 659.7 & 276.1 & 75.6 & 68.9 \\
\hline
\end{tabular}

(1a) $\widehat{\mu}=0.5, \widehat{\sigma}=2.3$ and $\widehat{\gamma}=6.4$. Subsample without extreme losses.

The two largest values of $X_{1}$ are excluded.

(1b) $\widehat{\mu}=0.7, \widehat{\sigma}=3.8$ and $\widehat{\gamma}=8.7$. Full sample.

(2a) $\widehat{\mu}=0.2, \widehat{\sigma}=1.2$ and $\widehat{\gamma}=0$. Subsample without extreme losses.

The two largest values of $X_{2}$ are excluded.

(2b) $\widehat{\mu}=0.5, \widehat{\sigma}=3.4$ and $\widehat{\gamma}=0$. Full sample.

(3a) $\widehat{\mu}=0.3, \widehat{\sigma}=0.3$ and $\widehat{\gamma}=2.6$. Subsample without extreme losses.

The two largest values of $X_{2}$ are excluded.

(3b) $\widehat{\mu}=0.3, \widehat{\sigma}=0.4$ and $\widehat{\gamma}=1.4$. Full sample.

(4a) $\widehat{\mu}=1.0, \widehat{\sigma}=3$ and $\widehat{\gamma}=5.9$. Subsample without extreme losses.

The two largest values of $X_{1}+X_{2}+X_{3}$ are excluded.

${ }^{(4 b)} \widehat{\mu}=1.4, \widehat{\sigma}=6.8$ and $\widehat{\gamma}=11.4$. Full sample.

Some comments related to outcome values for Cornish-Fisher approximations of the quantile-based risk measures should be made. According to the results, one could think that this kind of risk measurement corresponds to a conservative attitude for the two types of approximations shown in Table 4.2. The exception would be risk values obtained when $X_{2}$ is evaluated. 
Relevant differences are observed depending on the approximation finally used on right skewed data. Outcome values related to the second CornishFisher approximation (full sample) are drastically large when the data are severely right skewed distributed, as in the case of $X_{1}$ and $X_{1}+X_{2}+X_{3}$. These outcome values would be associated to a excessively conservative (unrealistic) attitude. If the first Cornish-Fisher approximation is considered, i.e. when sample statistics were estimated excluding extreme losses, a significant drop is observed although the outcome values for this approximation are still larger than those values associated with the empirical or the parametric distributions for right-skewed random variables. Note that only the two largest losses are not included in the sample estimates involving the first approximation. When the data are slightly right skewed distributed, as in the case of $X_{3}$, the two Cornish-Fisher approximations show a better performance. In other words, the Cornish-Fisher approximation should be used with certain caution when the data are severely right skewed distributed. Probably higher order moments should be taken into account. An important issue that arises from these results is the model risk. Even when the same risk measure is used, huge differences are observed depending on the hypothesis about the underlying distribution of the claim cost random variables. Let us assume that the regulator is focused on the VaR95\% for the aggregate cost $X_{1}+X_{2}+X_{3}$ as a measure of pure underwriting risk (without taking into account the premium paid by the policyholders). If it is supposed that the random variable is Pareto distributed, then the institution will need 5 thousands of euros for regulatory solvency purposes. The company should set aside almost 3.2 times this economic amount whether the underlying distribution is Student $t$ with 4 degrees of freedom. This topic is out of the scope of this chapter. The interested reader is addressed, for instance, to the study of Alexander and Sarabia [2012] which deals with VaR model risk or to the reference Aggarwal et al. [2016] suggested at the end of Chapter 1.

\subsection{Exercises}

1. Determine if the GlueVaR $\mathrm{R}_{99.5 \%, 95 \%}^{11 / 30,2 / 3}$ of a Normal distributed random variable $X$ with $\mathscr{N}\left(\mu=5, \sigma^{2}=16\right)$ satisfies the expressions related to the properties of Translation invariance, Positive homogeneity and Strictness.

2. Consider the Normal distributed random variable $X_{1}$ with $\mathscr{N}\left(\mu=5, \sigma^{2}=\right.$ 16) and the Normal distributed random variable $X_{2}$ with $\mathscr{N}\left(\mu=4, \sigma^{2}=\right.$ 
20). Show that:

- $\operatorname{VaR}_{\alpha}(Z)$ is equal to $\operatorname{VaR}_{\alpha}\left(X_{1}\right)+\operatorname{VaR}_{\alpha}\left(X_{2}\right)$ for any $\alpha$ in the case that $r_{x y}=1$, where $Z=X_{1}+X_{2}$ and $r_{x y}$ is the Pearson linear correlation coefficient.

- When $r_{x y}<1$, then $\operatorname{VaR}_{\alpha}(Z)<\operatorname{VaR}_{\alpha}\left(X_{1}\right)+\operatorname{VaR}_{\alpha}\left(X_{2}\right)$.

- Repeat the analysis for the GlueVaR $\mathrm{R}_{99.5 \%, 95 \%}^{1 / 20,1 / 8}$ risk measure.

3. Check that if $X$ is distributed as a Pareto $(X \sim \mathscr{G} \mathscr{P}(k, \sigma)$, with $k<0)$, the expression for $\operatorname{TVaR}_{\alpha}(X)$ shown in Table 1.3 may be obtained either:

- From Definition 1.9 (i.e, $\left.\operatorname{TVaR}_{\alpha}(X)=\frac{1}{1-\alpha} \int_{\alpha}^{1} \operatorname{VaR}_{\lambda}(X) d \lambda\right)$, or

- From the expression of $\operatorname{TVaR}_{\alpha}(X)$ as a distortion risk measure shown in Section 3.2 (i.e.

$$
\operatorname{TVaR}_{\alpha}(X)=\int_{-\infty}^{0}\left[\gamma_{\alpha}\left(S_{X}(x)\right)-1\right] d x+\int_{0}^{+\infty} \gamma_{\alpha}\left(S_{X}(x)\right) d x
$$

where $\gamma_{\alpha}$ is the distortion function displayed in Table 3.2).

Hint: Note that, in this case,

$$
\left(1-\frac{k}{\sigma} \operatorname{VaR}_{\alpha}(X)\right)^{1 / k}=S_{X}\left(\operatorname{VaR}_{\alpha}(X)\right)=1-\alpha .
$$

4. Obtain the RVaR risk measure equivalent to the GlueVaR $\mathrm{R}_{95 \%, 90 \%}^{0,1}$. 